\title{
Successful treatment with benralizumab in a patient with eosinophilic granulomatosis with polyangititis refractory to mepolizumab
}

\author{
Francesco Menzella, Carla Galeone, Giulia Ghidoni, Patrizia Ruggiero, Silvia Capobelli, Anna Simonazzi, \\ Chiara Catellani, Chiara Scelfo, Francesco Livrieri, Nicola Facciolongo \\ Pneumology Unit, Arcispedale Santa Maria Nuova, Azienda USL di Reggio Emilia IRCCS, Reggio Emilia, Italy
}

\begin{abstract}
Introduction: Eosinophilic granulomatosis with polyangiitis (EGPA) is characterized by necrotizing eosinophilic granulomatous inflammation that frequently involves the respiratory tract ( $90 \%$ of cases). Asthma in EGPA is systematically severe and often refractory to common treatment, it is corticosteroid resistant and can often anticipate the onset of systemic vasculitis by many years. A release of cytokines necessary for the activation, maturation and survival of eosinophils, such as IL-4, IL-5 and IL-13 occurs in the activated Th-2 phenotype. In particular, IL-5 level is high in active EGPA and its inhibition has become a key therapeutic target. Oral glucocorticoids (OCS) are effective treatment options but unfortunately, frequent relapses occur in many patients and they lead to frequent side effects. As for now, there are currently no official recommendations on doses and treatment schedules in the management of EGPA.

Case presentation: In this article, we describe the case of a man with EGPA, severe asthma and chronic rhinosinusitis with nasal polyps (CRSwNP), with poor asthma and CRSwNP control despite OCS and mepolizumab treatment. Respiratory and vasculitis symptoms improved markedly after therapeutic switch to benralizumab. During the treatment, in addition to clinical effects, we witnessed a depletion of blood eosinophils, as well as an improvement in both pulmonary function tests, CT scan and skin lesions present initially.

Conclusions: While there are many studies confirming the efficacy of benralizumab in EGPA, the most interesting aspect of our report is that efficacy was confirmed in a patient previously unresponsive to mepolizumab, known to be effective in EGPA.
\end{abstract}

Key words: Vasculitis; asthma; eosinophils; oral corticosteroids; biologics; IL-5.

Correspondence: Francesco Menzella, Pneumology Unit, Arcispedale Santa Maria Nuova, Azienda USL di Reggio Emilia IRCCS, 42123 Reggio Emilia, Italy. E-mail: francesco.menzella@ausl.re.it

Conflict of interest: FM has received research grants from AstraZeneca, Novartis and Sanofi; lecture fees and advisory board fees from AstraZeneca, Boehringer Ingelheim, Chiesi, GSK, Mundipharma, Angelini, Novartis, and Sanofi. The other authors declare no conflict of interest.

Availability of data and materials: All data underlying the findings are fully available.

Ethics approval and consent to participate: No ethical committee approval was required for this case report by the Department, because this article does not contain any studies with human participants or animals.

Consent for publication: A written informed consent for publication of their details was obtained from the patient involved in this study. The authors are available to share this form with the journal editorial office if requested. 


\section{Introduction}

Eosinophilic granulomatosis with polyangiitis (EGPA) is characterized by necrotizing eosinophilic granulomatous inflammation that frequently involves the respiratory tract ( $90 \%$ of cases). Another feature of this disease is the presence of a necrotizing vasculitis that mainly affects small and medium vessels and is associated with asthma and eosinophilia. In 2012, after the Chapel Hill international consensus conference [1], the name "Churg-Strauss syndrome" was replaced by EGPA [2]. The evolution of EGPA commonly follows three phases [3,4]: i) onset of asthma associated with rhinitis; ii) tissue eosinophilia; and iii) extrapulmonary eosinophil involvement with vasculitis. Asthma in EGPA is systematically severe and often refractory to common treatment [5], it is corticosteroid resistant and can often anticipate the onset of systemic vasculitis by many years [6,7]. Antineutrophil cytoplasmic antibodies (ANCA) are present in approximately $40 \%$ of EGPA patients $[8,9]$. ANCA play an important role in the diagnosis and in the pathogenesis of EGPA. These autoantibodies cause endothelial damage, as well as the infiltration of eosinophils, which are mechanisms underlying the typical histological damage. The pathogenetic effects of ANCA are due to their ability to activate neutrophils with consequent tissue and vascular damage [10,11]. A release of cytokines necessary for the activation, maturation and survival of eosinophils, such as IL-4, IL-5 and IL-13 occurs in the activated Th-2 phenotype [12,13]. In particular, IL-5 level is high in active EGPA and its inhibition has become a key therapeutic target as in severe eosinophilic asthma [14-16]. The increase in eosinophils has a cytotoxic and pro-coagulant effect that often determines not only pulmonary, but also cardiovascular and cerebrovascular organ damage $[17,18]$. The existence of a cross-talk between $\mathrm{T}$ lymphocytes and eosinophils has also been demonstrated. It is driven by a high concentration of IL-25, which induces T cells to produce cytokines stimulating the Th-2 and eosinophilic response, due to a positive feedback effect. The pathogenesis of EGPA is not due only to an increased Th-2 response [19]. The immune response is also amplified by endothelial and epithelial cells that produce chemokines, mainly eotaxin-3 (CCL-26) and chemokine-17 (CCL-17), which have a chemotaxis effect on eosinophils [20]. Eotaxin-3 is particularly elevated in patients with active EGPA and it significantly correlates with the level of eosinophils, total IgE and inflammation related parameters [21].

Oral glucocorticoids (OCS) reduce blood and tissue eosinophils by inducing apoptosis and inhibiting survival signaling pathways; they have long been regarded as the first-line treatment for EGPA [22]. Unfortunately, frequent relapses occur in many patients after dose tapering or despite high maintenance doses $[23,24]$. Prolonged therapies with OCS (or sometimes high doses for short periods) lead to severe and sometimes irreversible adverse effects, such as infections, osteoporosis, diabetes and secondary adrenal insufficiency $[25,26]$. Immunosuppressive agents are indicated as maintenance therapy and in the treatment of EGPA exacerbations [27]. Despite this use, some evidence indicates that immunosuppressive drugs do not lead to significant reductions in relapse rates [28]. As a result, there is a great need for truly effective treatment options which are burdened with fewer side effects. Mepolizumab, a fully humanized anti-IL-5 IgG1 monoclonal antibody (mAb), was evaluated in the MIRRA trial [29], at a dose of $300 \mathrm{mg} / 4$ weeks in patients with EGPA, demonstrating its superiority over standard treatment in terms of remission rate, time required for first relapse, reduction in OCS dose. However, a significant proportion of the patients in this study (approximately half) did not even achieve remission with mepolizumab co-administered with OCS. Anyway, the US Food and Drug Administration (FDA) approved mepolizumab $300 \mathrm{mg} / 4$ weeks for EGPA [30].
In addition to this, there are retrospective real-life studies confirming the efficacy of mepolizumab even at the dose of $100 \mathrm{mg}$ (approved for asthma) in EGPA during a remission phase [31,32]. Benralizumab, a humanized IgG1k afucosylated monoclonal antibody anti IL-5 receptor $\alpha$ chain, demonstrated efficacy in numerous studies, especially in real contexts, in terms of reduction of the average dose of OCS, a rapid depletion of blood eosinophils, a consistent improvement of respiratory symptoms and lung function in patients with EGPA [33-37]. In this article, we describe the case of a man with EGPA, severe asthma and chronic rhinosinusitis with nasal polyps (CRSwNP), with poor asthma control despite OCS and mepolizumab treatment. Respiratory symptoms improved markedly after therapeutic switch to benralizumab.

\section{Case presentation}

A 57-year-old man, non-smoker, came to our observation. He worked as an industrial machinery mechanic. The patient was not exposed to inhalation of chemicals and there were no changes in respiratory symptoms away from the workplace. In May 2018, due to persistent dry cough, dyspnea and wheezing he was sent by his general practioner for a respiratory clinical assessment. On the physical examination of the chest, rhonchi and wheezing were detected. Respiratory function tests showed moderate bronchial obstruction (Forced expiratory volume in 1 second $\left(\mathrm{FEV}_{1}\right) 62 \%$ of predicted) with a significant response to the bronchodilation test ( + $18 \% ;+480 \mathrm{ml}$ ). Skin prick tests were negative for inhalant allergens while blood tests showed eosinophilia (790 cells/ul, 8\%). Body mass index (BMI) was 24. Therefore, bronchial asthma was diagnosed and therapy was started with high dose salmeterol / fluticasone, salbutamol spray as needed and prednisone $25 \mathrm{mg} 2$ tablets a day for 5 days, with gradual reduction. A computed tomography (CT) scan of the paranasal sinuses showed mucosal thickening in the right maxillary sinus with osteomeatal engagement and initial mucosal thickening in the left maxillary sinus and both frontal sinuses. Sinonasal Outcome Test 22 (SNOT-22) score was 51.5. For persistence of cough and bronchospasm, the patient referred to an otolaryngologist, who evidenced mucosal secretion with post-nasal drip, edema of the right meatus and bilateral polyposis. He also hypothesized gastroesophageal reflux due to typical physical examination findings, including arytenoid erythema and edema. An esophagogastroduodenoscopy (EGD) was then performed, revealing grade A reflux esophagitis. A therapy with a proton pump inhibitor (PPI) was then set up, with esomeprazole 40 mg per day. Then, the patient repeated a pulmonology examination in July 2018 due to poor improvement in respiratory symptoms. An assessment of medication adherence had been made, and was appropriate. Consequently, the therapy was modified by stopping salmeterol/fluticasone and starting high dose of beclometasone/formoterol spray and adding tiotropium bromide. Prednisone $25 \mathrm{mg}$ per day was also included due to recurrence of asthma symptoms after an attempted withdrawal of OCS. Blood tests performed in September 2018 showed eosinophilia (4790 cells/ul, 27\%), Antineutrophil myeloperoxidase cytoplasmic antibodies (MPO-ANCA) level was 17.9, whilst proteinase 3 (PR3ANCA), antinuclear Antibodies (ANA), extractable nuclear antigen (ENA) were negative. Total immunoglobulin E ( $\mathrm{IgE}$ ) were 113 $\mathrm{IU} / \mathrm{mL}($ v.n. $<100)$ and fractional exhaled nitric oxide (FeNO) value (ppb) 43. Allergic bronchopulmonary aspergillosis and intestinal parasites infection were ruled out. The patient then underwent a high-resolution chest CT scan, that showed parenchymal centrilobular nodules and the presence of multiple, faded, bilateral ground glass areas. Skin lesions on the back of the hands were detected and they were compatible with vasculitis according to the 
dermatologist's diagnosis and a skin biopsy showing leukocytoclastic vasculitis with several eosinophils in the dermal infiltrate. In suspicion of vasculitis, the patient underwent a fibrobronchoscopy with bronchoalveolar lavage (BAL) and transbronchial biopsies; histological findings showed necrotizing small vessel vasculitis associated with eosinophil infiltrates and perivascular and extravascular granulomas. BAL demonstrated macrophages and rare lymphocytes with neutrophilic granulocytes and alveolar eosinophilia (35\%). Doppler echocardiography was normal. The patient was seen by a rheumatologist, who confirmed the diagnosis of EGPA but did not indicate add-on immunosuppressive therapy with azathioprine/mycophenolate or rituximab/cyclophosphamide, also considering the absence of mononeuritis multiplex in our patient. Mononeuritis multiplex predicts the need for immunosuppressive or immunomodulatory drugs for EGPA, and its absence reduces the need for these add on drugs burdened with important side effects [38]. On January 2019, due to the persistence of poor asthma control with the need for daily therapy with OCS, after a pulmonology evaluation, the patient began therapy with mepolizumab $100 \mathrm{mg}$ subcutaneously (SC). Four months later, asthma symptoms persisted unchanged and, in an attempt to improve respiratory symptoms, the patient underwent Functional Endoscopic Sinus Surgery (FESS) for CRSwNP. The patient had a temporary improvement in nasal and bronchial symptoms, with a SNOT-22 score of 44.3, compared to the baseline of 51.5. Subsequently he returned to previous symptoms. Blood eosinophils were 280 cells/ul (3\%) and FeNO was 18 ppb. In August 2019 a new pulmonology examination was carried out, due to suboptimal asthma control without the possibility of reducing OCS dosage. Therefore, it was decided to discontinue mepolizum$\mathrm{ab}$ after seven administrations due to lack of efficacy. After a 3month washout period the patient started benralizumab $30 \mathrm{mg} \mathrm{sc}$ once every 4 weeks for the first three doses, then once every 8 weeks. We excluded mepolizumab $300 \mathrm{mg}$ once every 4 weeks because it was not approved in Europe for EGPA therapy yet and our local ethics committee hardly grants approval to the higher dose since an alternative therapeutic option such as benralizumab is available. In contrast, the standard dose $(30 \mathrm{mg})$ of benralizumab was very effective and in the literature there are studies that confirm the great efficacy of benralizumab in EGPA thanks to profound antieosinophilic effect in different tissues. These could be potential advantages of this biologic in the treatment of EGPA [39]. Respiratory symptoms progressively improved after just 3 months. The patient was reassessed by a respiratory physician in March 2020, recording excellent asthma control and an improvement in symptoms related to CRSwNP (SNOT-22 score improved to 34.6). The dosage of prednisone was then reduced to $8 \mathrm{mg}$ per day, then subsequently reduced to $4 \mathrm{mg}$ per day and finally withdrawn 6 months after starting benralizumab therapy (Table 1). Regarding blood tests, an important reduction in MPO-ANCA and blood eosinophils was found ( 0 cells/ul, $0.1 \%$ ). FeNO value was 13 ppb. Since the start of benralizumab therapy 12 months before, the patient has not presented any asthma exacerbation (Figure 1) and respiratory function tests have improved, returning to normal levels $\left(\mathrm{FEV}_{1}\right.$ pred., FVC 91\%pred., FEV $/$ FVC 105\%) (Table 2 and Figure 2). High-resolution chest CT no longer showed groundglass opacities and centrilobular nodules present at baseline control and also the skin lesions on the back of the hands present before the start of biological therapy had disappeared. Another fundamental aspect was the absence of side effects with the use of benralizumab during the whole observation period.

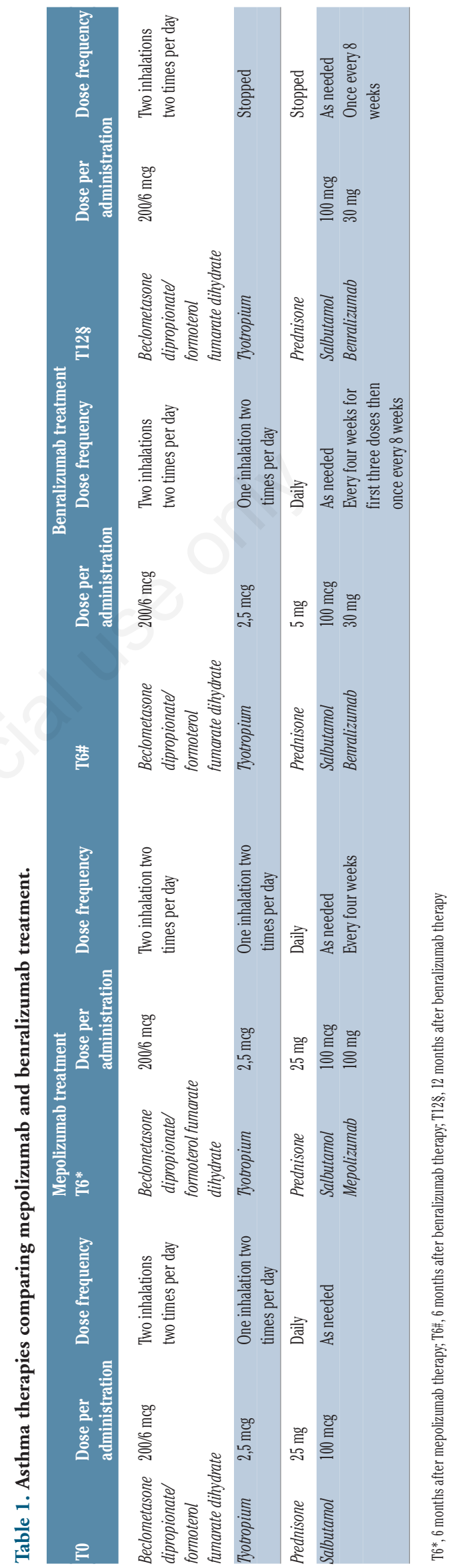




\section{Discussion and conclusions}

Despite the great progresses made in recent years in the knowledge of the pathogenesis of EGPA, management and therapy of this disease remain difficult tasks. Patients often suffer from refractory or relapsing conditions despite immunosuppressant and corticosteroid therapies. These drugs are also burdened with important side effects. After the excellent results obtained with mepolizumab in the treatment of severe eosinophilic asthma (SEA), the anti-IL5 biologic has also been studied in EGPA $[40,41]$. Mepolizumab was approved by the FDA at a dose of 300 mg SC every 4 weeks after the results of just one randomized clinical trial (RCT) [29]. Nevertheless, in some real-life studies mepolizumab was given at the dose 150 of $100 \mathrm{mg}$ every 4 weeks, the dose approved for the treatment of SEA [31,42]. In the DREAM pivotal study, a comparable clinic efficacy was demonstrated with three intravenous (IV) doses of mepolizumab: 75, and $750 \mathrm{mg}$, every 4 weeks; in the subsequent MENSA trial, the effective dose was $100 \mathrm{mg}$ SC every 4 weeks [40,41]. The first studies on the use of mepolizumab in EGPA reported clinical efficacy at the $750 \mathrm{mg}$ IV dose [43-46]. Considering these findings, we treated our patient with the lowest dose of mepolizumab, but without obtaining positive clinical results. As the indication of mepolizum$\mathrm{ab}$ for EGPA at a dose of $300 \mathrm{mg}$ has not been approved in Europe yet, it is usually necessary to obtain approval from ethics committees to treat patients. Therefore, we decided to carry out a therapeutic switch to benralizumab, given its potent effect on reducing eosinophils, which are the key of EGPA pathogenesis. The high affinity of benralizumab to the $\alpha$ chain of IL-5R blocks activation
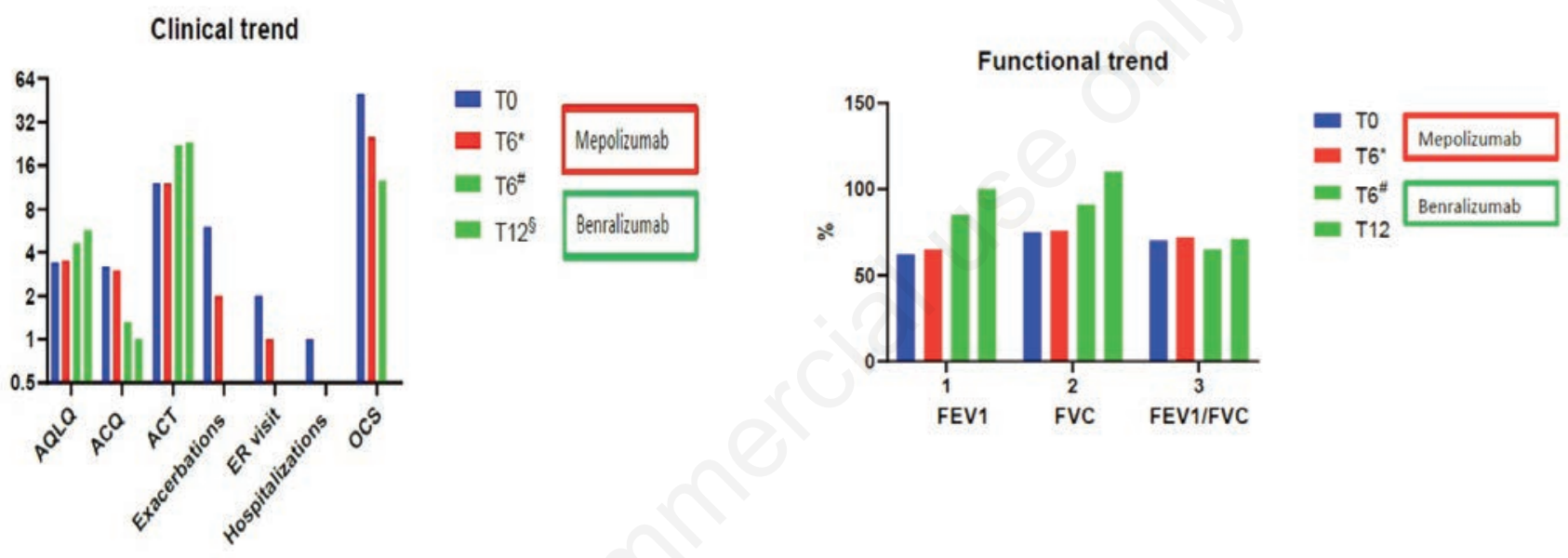

Figure 1. Clinical trend of the patient.

Figure 2. Functional trend of the patient.

Table 2. Clinical outcomes comparing baseline, mepolizumab and benralizumab treatment.

\begin{tabular}{|c|c|c|c|c|}
\hline & & Mepolizumab therapy & & \\
\hline & T0 & $\mathrm{T}^{*}$ & T6\# & $\mathrm{T} 12 \S$ \\
\hline AQLQ (score) & 3.4 & 3.5 & 4.6 & 5.7 \\
\hline ACQ (score) & 3.2 & 3 & 1.3 & 1 \\
\hline ACT (score) & 12 & 12 & 22 & 23 \\
\hline Exacerbations (n) & 6 & 2 & 0 & 0 \\
\hline ER visit (n) & 2 & 1 & 0 & 0 \\
\hline Hospitalizations (n) & 1 & 0 & 0 & 0 \\
\hline Hospitalizations' duration (days) & 7 & 0 & 0 & 0 \\
\hline Days missed from work & 33 & 9 & 0 & 0 \\
\hline OCS daily dose (prednisone, mg) & 50 & 25 & 12.5 & 0 \\
\hline FEV1 (\%) & 62 & 65 & 85 & 100 \\
\hline FEV1 (L) & 2.03 & 2.1 & 2.77 & 3.47 \\
\hline FVC (\%) & 75 & 76 & 91 & 110 \\
\hline FVC (L) & 2.9 & 2.9 & 4.2 & 4.86 \\
\hline FEV1/FVC (\%) & 70 & 72 & 65 & 71 \\
\hline
\end{tabular}

T6* 6 months after mepolizumab therapy; T6\#, 6 months after benralizumab therapy; T12§, 12 months after benralizumab therapy. 
and signal transduction. Furthermore, the afucosylation increases the function of cell-mediated cytotoxicity dependent on antibodies (ADCC), making this biological drug able to reduce the number of circulating eosinophils between 90 and $100 \%$ in various tissues [47]. This peculiar mechanism of action could represent an advantage in EGPA. As for mepolizumab, various studies in real world settings confirmed efficacy of benralizumab not only in SEA, but also in EGPA [33-36,39]. Data from an ongoing RCT suggesting the efficacy and OCS sparing effect of benralizumab (NCT04157348) are not available yet [48]. Another ongoing RCT (NCT03010436) is recruiting patients with the purpose to evaluate the efficacy and safety of benralizumab $30 \mathrm{mg}$ compared to mepolizumab $300 \mathrm{mg}$ administered by SC injection (SC) in patients with relapsed or refractory EGPA on OCS therapy with or without stable immunosuppressive therapy [49]. A recent prospective open-label pilot study in a small number of EGPA patients treated with benralizumab $30 \mathrm{mg}$ sc showed that the drug was well tolerated and it allowed to reduce OCS and EGPA relapses [34]. In our OCS and mepolizumab refractory EGPA patient, benralizumab confirmed great clinical efficacy while maintaining an excellent safety profile and also allowing complete weaning from OCS. During the course of treatment, in addition to the positive clinical effects, we witnessed a rapid and stable depletion of blood eosinophils and MPO-ANCA, as well as an improvement in both pulmonary function tests and $\mathrm{CT}$ scan. Consequently, there was no need for initiating therapy with mepolizumab $300 \mathrm{mg}$ every 4 weeks, probably more effective than the lower dose but also burdened with much higher pharmacoeconomic costs, already high at the $100 \mathrm{mg}$ dose [50].

The present study shows that benralizumab was effective in obtaining a great improvement in our patient outcomes, with positive results just after a few weeks, that were mainteined after a year, thanks to its "aggressive" effect of tissue depletion of eosinophils. These excellent effects are even more remarkable as they were obtained in a patient unresponsive to a previous treatment with an anti-IL5 agent, known to be effective in EGPA. It is important to note that our patient had some characteristics that allowed better results with benralizumab, notably high blood eosinophilia, CRSwNP, impaired respiratory function and frequent exacerbations. These aspects could guide the choice of this therapeutic option in EGPA, as it already happens in SEA as well. Nevertheless, clinical studies on larger populations with selected patients and head-to-head comparisons between anti-IL5 biologics are needed.

\footnotetext{
Abbreviations

EGPA: Eosinophilic granulomatosis with polyangiitis;

ANCA: Antineutrophil cytoplasmic antibodies;

OCS: Oral glucocorticoids;

FDA: US Food and Drug Administration;

CRSwNP: chronic rhinosinusitis with nasal polyps;

$\mathrm{FEV}_{1 \text { : }}$ Forced expiratory volume in 1 second;

BMI: body mass index;

CT: computed tomography scan,

EGD: esophagogastroduodenoscopy;

PPI: proton pump inhibitor;

PR3-ANCA: proteinase 3 antineutrophil cytoplasmic antibodies;

ANA: antinuclear antibodies;

ENA: extractable nuclear antigen;

IgE: Immunoglobulin E;

FeNO: fractional exhaled nitric oxide;

FESS: functional endoscopic sinus surgery;

SEA: severe eosinophilic asthma;
}

RCT: randomized clinical trial;

IV: intravenous;

ADCC: cell-mediated cytotoxicity dependent on antibodies.

\section{References}

1. Jennette JC, Falk RJ, Bacon PA, Basu N, Cid MC, Ferrario F, et al. 2012 revised International Chapel Hill Consensus Conference Nomenclature of Vasculitides. Arthritis Rheum 2013;65:1-11.

2. Cordier JF, Cottin V, Guillevin L, Bel E, Bottero P, Dalhoff K, et al. Eosinophilic granulomatosis with polyangiitis (ChurgStrauss). Presse Med 2013;42:507-10.

3. Cottin V, Cordier JF. Eosinophilic pneumonia. In: Mason RJ, Ernst JD, King TE Jr, et al., editors. Murray and Nadel's Textbook of Respiratory Medicine. 6th Ed. Philadelphia, Elsevier Saunders, 2016; p. 1221-1242.

4. Mahr A, Moosig F, Neumann T, Szczeklik W, Taillé C, Vaglio A, et al. Eosinophilic granulomatosis with polyangiitis (ChurgStrauss): evolutions in classification, etiopathogenesis, assessment and management. Curr Opin Rheumatol 2014;26:16-23.

5. Tsurikisawa N, Tsuburai T, Saito H, Morita S, Horiguchi Y, Mitomi $\mathrm{H}$, et al. A retrospective study of bronchial hyperresponsiveness in patients with asthma before the onset of Churg-Strauss syndrome. Allergy Asthma Proc 2007;28:33643.

6. Lanham JG, Elkon KB, Pusey CD, Hughes GR. Systemic vasculitis with asthma and eosinophilia: a clinical approach to the Churg-Strauss syndrome. Medicine (Baltimore) 1984;63:6581.

7. Guillevin L, Cohen P, Gayraud M, Lhote F, Jarrousse B, Casassus P. Churg-Strauss syndrome. Clinical study and longterm follow-up of 96 patients. Medicine (Baltimore) 1999;78:26-37.

8. Kiene M, Csernok E, Müller A, Metzler C, Trabandt A, Gross WL. Elevated interleukin-4 and interleukin-13 production by $\mathrm{T}$ cell lines from patients with Churg-Strauss syndrome. Arthritis Rheum 2001;44:469-73.

9. Dunogué B, Pagnoux C, Guillevin L. Churg-strauss syndrome: clinical symptoms, complementary investigations, prognosis and outcome, and reatment. Semin Respir Crit Care Med 2011;32:298-309.

10. Falk RJ, Terrell RS, Charles LA, Jennette JC. Anti-neutrophil cytoplasmic autoantibodies induce neutrophils to degranulate and produce oxygen radicals in vitro. Proc Natl Acad Sci USA 1990;87:4115-9.

11. Xiao H, Heeringa P, Hu P, Liu Z, Zhao M, Aratani Y, et al. Antineutrophil cytoplasmic autoantibodies specific for myeloperoxidase cause glomerulonephritis and vasculitis in mice. J Clin Invest 2002;110:955-63.

12. Jakiela B, Sanak M, Szczeklik W, Sokolowska B, Plutecka H, Mastalerz L, et al. Both Th2 and Th17 responses are involved in the pathogenesis of Churg-Strauss syndrome. Clin Exp Rheumatol 2011;29:S23-34.

13. Jakiela B, Szczeklik W, Plutecka H, Sokolowska B, Mastalerz L, Sanak M, et al. Increased production of IL-5 and dominant Th2-type response in airways of Churg-Strauss syndrome patients. Rheumatology 2012;51:1887-93.

14. Moosig F, Gross WL, Herrmann K, Bremer JP, Hellmich B. Targeting interleukin-5 in refractory and relapsing ChurgStrauss syndrome. Ann Intern Med 2011;155:341-3.

15. Terrier B, Bièche I, Maisonobe T, Laurendeau I, Rosenzwajg M, Kahn JE, et al. Interleukin-25: a cytokine linking eosinophils and adaptive immunity in Churg-Strauss syn- 
drome. Blood 2010;116:4523-31.

16. Vaglio A, Moosig F, Zwerina J. Churg-Strauss syndrome: update on pathophysiology and treatment. Curr Opin Rheumatol 2012;24:24-30.

17. Mahr A, Guillevin L, Poissonnet M, Aymé S. Prevalences of polyarteritis nodosa, microscopic polyangiitis, Wegener's granulomatosis, and Churg-Strauss syndrome in a French urban multiethnic population in 2000: A capture-recapture estimate. Arthritis Care Res 2004;51:92-9.

18. Watts RA, Lane SE, Bentham G, Scott DG. Epidemiology of systemic vasculitis: a ten-year study in the United Kingdom. Arthritis Rheum 2000;43:414-9.

19. Ramentol-Sintas M, Martínez-Valle F, Solans-Laqué R. ChurgStrauss Syndrome: an evolving paradigm. Autoimmun Rev 2012;12:235-40.

20. Zwerina J, Bach C, Martorana D, Jatzwauk M, Hegasy G, Moosig F, et al. Eotaxin-3 in Churg-Strauss syndrome: a clinical and immunogenetic study. Rheumatology 2011;50:1823-7.

21. Churg A. Recent advances in the diagnosis of Churg-Strauss syndrome. Mod Pathol 2001;14():1284-1293.

22. Fulkerson PC, Rothenberg ME. Targeting eosinophils in allergy, inflammation and beyond. Nat Rev Drug Discov 2013;12:117-29.

23. Comarmond C, Pagnoux C, Khellaf M, Cordier JF, Hamidou M, Viallard JF, et al. Eosinophilic granulomatosis with polyangiitis (Churg-Strauss): clinical characteristics and longterm follow-up of the 383 patients enrolled in the French Vasculitis Study Group cohort. Arthritis Rheum 2013;65:27081.

24. Samson M, Puechal X, Devilliers H, Ribi C, Cohen P, Stern M, et al. Long-term outcomes of 118 patients with eosinophilic granulomatosis with polyangiitis (Churg-Strauss syndrome) enrolled in two prospective trials. J Autoimmun 2013;43:60-9.

25. Daugherty J, Lin X, Baxter R, Suruki R, Bradford E. The impact of long-term systemic glucocorticoid use in severe asthma: a UK retrospective cohort analysis. J Asthma 2018;55:651-8.

26. Strehl C, Bijlsma JW, de Wit M, Boers M, Caeyers N, Cutolo $\mathrm{M}$, et al. Defining conditions where long-term glucocorticoid treatment has an acceptably low level of harm to facilitate implementation of existing recommendations: viewpoints from an EULAR task force. Ann Rheum Dis 2016;75:952-7.

27. Groh M, Pagnoux C, Baldini C, Bel E, Bottero P, Cottin V, et al. Eosinophilic granulomatosis with polyangiitis (ChurgStrauss) (EGPA) Consensus Task Force recommendations for evaluation and management. Eur J Intern Med 2015;26:54553.

28. Puechal X, Pagnoux C, Baron G, Quémeneur T, Néel A, Agard $\mathrm{C}$, et al. Adding azathioprine to remission-induction glucocorticoids for eosinophilic granulomatosis with polyangiitis (Churg-Strauss), microscopic polyangiitis, or polyarteritis nodosa without poor prognosis factors: a randomized, controlled trial. Arthritis Rheumatol 2017;69:2175-86.

29. Wechsler ME, Akuthota P, Jayne D, Khoury P, Klion A, Langford CA, et al. Mepolizumab or placebo for eosinophilic granulomatosis with polyangiitis. N Engl J Med 2017;376:1921-32.

30. U.S. Food and Drug Administration. Nucala prescribing information. Accessed: January 1, 2021. Available from: www.accessdata.fda.gov.

31. Caminati M, Crisafulli E, Lunardi C, Micheletto C, Festi G, Maule M, et al. Mepolizumab $100 \mathrm{mg}$ in severe asthmatic patients with EGPA in remission phase. J Allergy Clin Immunol Pract 2021;9:1386-8.
32. Vultaggio A, Nencini F, Bormioli S, Vivarelli E, Dies L, Rossi $\mathrm{O}$, et al. Low-dose mepolizumab effectiveness in patients suffering from eosinophilic granulomatosis with polyangiitis. Allergy Asthma Immunol Res 2020;12:885-93.

33. Coppola A, Flores KR, De Filippis F. Rapid onset of effect of benralizumab on respiratory symptoms in a patient with eosinophilic granulomatosis with polyangiitis. Respir Med Case Rep 2020;30:101050.

34. Guntur VP, Manka LA, Denson JL, Dunn RM, Dollin YT, Gill $\mathrm{M}$, et al. Benralizumab as a steroid-sparing treatment option in eosinophilic granulomatosis with polyangiitis. J Allergy Clin Immunol Pract 2021;9:1186-93.

35. Miyata Y, Inoue H, Homma T, Tanaka A, Sagara H. Efficacy of benralizumab and clinical course of Igg4 on eosinophilic granulomatosis with polyangiitis. J Investig Allergol Clin Immunol 2020;0. Online ahead of print.

36. Nanzer AM, Dhariwal J, Kavanagh J, Hearn A, Fernandes M, Thomson L, et al. Steroid-sparing effects of benralizumab in patients with eosinophilic granulomatosis with polyangiitis. ERJ Open Res 2020;6:00451-2020.

37. Padoan R, Chieco Bianchi F, Marchi MR, Cazzador D, Felicetti M, Emanuelli E, et al. Benralizumab as a glucocorticoid-sparing treatment option for severe asthma in eosinophilic granulomatosis with polyangiitis. J Allergy Clin Immunol Pract 2020;8:3225-7.

38. Samson M, Puéchal X, Devilliers H, Ribi C, Cohen P, Bienvenu B, et al. Mononeuritis multiplex predicts the need for immunosuppressive or immunomodulatory drugs for EGPA, PAN and MPA patients without poor prognosis factors. Autoimmun Rev 2014;13:945-53.

39. Martínez-Rivera C, Garcia-Olivé I, Urrutia-Royo B, Basagaña-Torrento M, Rosell A, Abad J. Rapid effect of benralizumab in exacerbation of severe eosinophilic asthma associated with eosinophilic granulomatosis with polyangiitis. BMC Pulm Med 2021;21:35.

40. Pavord ID, Korn S, Howarth P, Bleecker ER, Buhl R, Keene $\mathrm{ON}$, et al. Mepolizumab for severe eosinophilic asthma (DREAM): a multicentre, double-blind, placebo-controlled trial. Lancet 2012;380:651-9.

41. Ortega HG, Liu MC, Pavord ID, russelle GG, FitzGerald JM, Chetta A, et al. Mepolizumab treatment in patients with severe eosinophilic asthma. N Engl J Med 2014;371:1198-207.

42. Vergles M, Matković Z, Lalić K, Trkanjec JT, Tudorić N. Mepolizumab as a glucocorticoid-sparing agent in eosinophilic granulomatosis with polyangiitis (EGPA): is a lower dose sufficient? J Asthma 2020;1-5. Online ahead of print.

43. Kahn JE, Grandpeix-Guyodo C, Marroun I, Marroun I, Catherinot E, Mellot F, et al. Sustained response to mepolizumab in refractory Churg-Strauss syndrome. J Allergy Clin Immunol 2010;125:267-70.

44. Kim S, Marigowda G, Oren E, Israel E, Wechsler ME. Mepolizumab as a steroid-sparing treatment option in patients with Churg-Strauss syndrome. J Allergy Clin Immunol 2010;125:1336-43.

45. Moosig F, Gross WL, Herrmann K, Bremer JP, Hellmich B. Targeting interleukin-5 in refractory and relapsing ChurgStrauss syndrome. Ann Intern Med 2011;155:341-3.

46. Herrmann K, Gross WL, Moosig F. Extended follow-up after stopping mepolizumab in relapsing/refractory Churg-Strauss syndrome. Clin Exp Rheumatol 2012;30:S62-5.

47. Menzella F, Biava M, Bagnasco D, Galeone C, Simonazzi A, Ruggiero $\mathrm{P}$, et al. Efficacy and steroid-sparing effect of benralizumab: has it an advantage over its competitors? Drugs Context 2019;8:212580. 
48. ClinicalTrials.gov [Internet]. Bethesda (MD): National Library of Medicine (US). 2019 Nov 8. Identifier NCT04157348, Efficacy and Safety of Benralizumab in EGPA Compared to Mepolizumab. (MANDARA). Accessed 3 January 2021. Available from: https://www.clinicaltrials.gov/ct2/show/ NCT04157348

49. ClinicalTrials.gov [Internet]. Bethesda (MD): National Library of Medicine (US). 2019 Nov 8. Identifier NCT03010436,
Benralizumab in the Treatment of Eosinophilic Granulomatosis With Polyangiitis (EGPA) Study (BITE). Accessed 3 January 2021. Available from: https://www.clinicaltrials.gov/ct2/show/NCT03010436

50. Tan LE, Tan WHG, Aziz MIA, Aziz MIA, Koh MS, Tay TR, et al. Assessing the cost-effectiveness of mepolizumab as add-on therapy to standard of care for severe eosinophilic asthma in Singapore. J Asthma 2020;1-11.

Received for publication: 5 May 2021.. Accepted for publication: 15 June 2021.

This work is licensed under a Creative Commons Attribution-NonCommercial 4.0 International License (CC BY-NC 4.0).

(C) Copyright: the Author(s), 2021

Licensee PAGEPress, Italy

Multidisciplinary Respiratory Medicine 2021; $16: 779$

doi:10.4081/mrm.2021.779 